Leitartikel

\title{
Kaufuntersuchung - die ständige Herausforderung. Brauchen wir einen erweiterten Standard?
}

\author{
H. D. Lauk
}

Es ist nun 15 Jahre her, dass sich interessierte Tierärzte und Juristen zusammengesetzt haben, um in Abstimmung mit der damaligen Bundestierärztekammer den Standard der Kaufuntersuchung zu fixieren und ein entsprechendes Formular zusammenzustellen, das als Vertrag und Protokoll dienen sollte. Man einigte sich auf den „Vertrag über die Untersuchung eines Pferdes" der in der Folgezeit bis heute seinen Zweck erfüllt hat und von der Kollegenschaft und den Gerichten als verbindliche Richtlinie anerkannt wurde. Doch die Zeit hat für die Praxis und den Pferdehandel Veränderungen mit sich gebracht. Die tierärztliche Diagnostik und der allgemeine tierärztliche Kenntnisstand haben sich verbessert, der Pferdekäufer ist anspruchsvoller und die Pferde sind teverer geworden. Nachdem nun auch das Schuldrecht geändert wurde scheint es an der Zeit, den Vertrag zu überarbeiten und den veränderten Anforderungen anzupassen.

Jede einzelne Kaufuntersuchung stellt für uns hinsichtlich der Aktivierung unserer Wissensressourcen und angesichts des forensischen Hintergrunds eine neve Herausforderung dar. In der Kaufuntersuchung bündeln sich all unsere diagnostischen Fähigkeiten, unsere Kenntnisse und vor allem unsere praktische Erfahrung. Innerhalb eines kurzen Zeitraums und mit eingeschränktem Aufwand müssen wir ein Maximum an diagnostischer Information erzielen. Der Diagnostik folgt nicht wie in der kurativen Praxis die Behandlung, sondern die Bewertung, die Beurteilung und die prognostische Einschätzung. Aus der Summe verschiedener Befunde und Eindrücke eines Pferdes folgt dann die Beratung des Auftraggebers, mit der wir ein hohes Maß an Verantwortung und auch Haftung übernehmen. Meist wünscht der Käufer nicht nur die Untersuchung und Bewertung der Befunde, sondern er erwartet vor allem Hilfe bei der Kaufentscheidung, in der er uns meist weitgehend einbindet. Und schon immer war es so, dass er, traten später gesundheitliche Probleme mit seinem Pferd auf, nur allzu leicht geneigt war, sich beim Tierarzt schadlos zu halten. Gerade hierzulande hat sich diesbezüglich eine besondere Streitkultur entwickelt und viele Stimmen äußern sich dahingehend, dass diese Kultur unter dem neven Schuldrecht weitere Blüten treiben wird.

\section{Einschätzung des Auftraggebers}

Erhalten wir den Auftrag zur Kaufuntersuchung, so ist zunächst die Erfahrung des Auftraggebers einzuschätzen. Danach richten sich Art und Umfang unserer Aufklärungspflicht. Handelt es sich um den Verkäufer, dann ist meist davon auszugehen, dass dieser um die Rahmenbedingungen von Pferdehandel und Kaufuntersuchung weiß und entsprechend können wir uns bei der Aufklärung kürzer fassen als dies der Fall ist, wenn es sich um einen unerfahrenen Käufer handelt. Je weniger dieser mit der
Materie vertraut ist, desto umfangreicher hat die Aufklärung über Möglichkeiten und Grenzen der Kaufuntersuchung, über die einzelnen Untersuchungsschritte, Risiken möglicher Eingriffe und über die Befunde auszufallen. Natürlich gibt es auch erfahrene Käufer, in der Mehrzahl der Fälle ist der Auftraggeber aber als Laie anzusehen. In jedem Fall haben wir durch angemessene Aufklärung beide Parteien auf die Bedingungen der Kaufuntersuchung vorzubereiten.

\section{Allgemeine Aufklärung}

Steht die Kaufuntersuchung für den Käufer an, dann ist dessen Entscheidung für ein bestimmtes Pferd nach seinen Kriterien meist schon gefallen und es liegt am Tierarzt aus veterinärmedizinischer Sicht zu- oder abzuraten. Meist ist dem Käufer, besonders aber der Käuferin, das Pferd in kürzester Zeit schon ans Herz gewachsen und eine mögliche Befunderhebung wird zunächst als Störung der positiven Befindlichkeit empfunden. An dieser Stelle ist oft Sensibilität aber auch eine klare Linie gefragt. Jeder Pferdekauf beinhaltet ein mehr oder weniger großes Risiko, das auch durch unsere Untersuchung nicht gänzlich verhindert, sondern allenfalls reduziert werden kann. Diese Risikoreduzierung ist unsere Aufgabe, und für diese Leistung werden wir bezahlt und dafür haften wir letztendlich. Das Risiko des Auftraggebers, bisher meist des Käufers, künftig vermutlich mehr des Verkäufers, ist also eine gewisse Zeit lang auch das unsere. Das bedeutet, dass wir mit unserer Tätigkeit nicht nur den Auftraggeber bestmöglich bedienen, sondern auch uns selbst bestmöglich vor möglichen späteren Ansprüchen schützen müssen.

Wir haben den Käufer zu beraten, ob das Pferd für die von ihm geplante Verwendung geeignet erscheint. Um diese Beratung zu gewährleisten, müssen wir eine möglichst gründliche Untersuchung durchführen. Es gibt jedoch kein fehlerfreies Pferd. Leicht findet sich im Einzelfall ein Grund, vom Kauf eines bestimmten Pferdes abzuraten. Übertriebene Vorsicht aus Angst vor möglichen Haftungsansprüchen macht jedoch keinen Sinn und dient letztendlich niemandem. Sie verärgert Käufer und Verkäufer und führt dazu, dass wir, wenn sich dies wiederholt und herumspricht, mit Kaufuntersuchungen nicht wieder betraut werden. Zwar ist jede Abweichung von der Norm zu erkennen, aufzuzeichnen, zu bewerten und der Käufer davon in Kenntnis zu setzen, aber letztendlich ist ein praktikabler Kompromiss zu finden, der in Hinsicht auf die geplante Verwendung des Pferdes angemessen ist. Der Käufer wünscht nicht nur die Untersuchung, sondern letztendlich unsere Meinung zu dem Pferd und insbesondere unseren Rat hinsichtlich der künftigen Entwicklung möglicher besonderer Befunde. 


\section{Prognose}

Gerade hier aber liegen die Grenzen, denn die Entwicklung der Gesundheit des Pferdes vorauszusagen ist grundsätzlich nicht möglich. Aus diesem Grunde dürfen prognostische Äußerungen nicht oder nur mit der größten Zurückhaltung gegeben werden. Die objektiven Grundlagen für die Untersuchung und Beratung ist der Zustand des Pferdes zum Zeitpunkt der Untersuchung. Der Auftraggeber kann nicht oft genug daran erinnert werden. Wird das Gesamturteil über das Pferd gefällt, dann sind alle Befunde insgesamt zu betrachten. Entscheidend ist letztendlich, wie alt das Pferd ist und wie es genutzt werden soll. Es ist durchaus denkbar, dass ein Pferd für den einen Käufer geeignet ist, für den anderen jedoch nicht. Zu diskutieren ist auch die Frage, ob das Pferd ggf. versichert werden soll und wie es sich später wieder verkaufen lässt. All dies ist mit dem Auftraggeber zu erörtern und nach positiver Kaufuntersuchung ist die Wirksamkeit des Kaufes ggf. auch von der Annahme des Pferdes durch die Versicherung abhängig.

\section{Vollständige Untersuchung und Protokollierung}

Um die Qualität der Untersuchung zu gewährleisten und zu unserer eigenen Sicherheit ist vom Grundsatz der vollständigen Untersuchung unter keinen Umständen abzurücken. Man wird immer wieder und mit ganz unterschiedlicher Intention mit dem Wunsch des Auftraggebers, ob Käufer oder Verkäufer, konfrontiert, die Untersuchung doch abzukürzen oder Teile wegzulassen. Manchmal mag man auch selbst dazu verführt sein, unter Zeitdruck etwas zügiger zu untersuchen. Diese Nachlässigkeiten sind gefährlich und oft genug der erste Schritt zum Scheitern vor Gericht. Auch gilt bei der Kaufuntersuchung, wie bei der kurativen Tätigkeit, dass was nicht schriftlich fixiert ist, in den Augen des Gerichts nicht durchgeführt worden ist. In solchen Fällen tritt die Beweislastumkehr ein und für diesen Ernstfall muss der Tierarzł auch über zuverlässige Zeugen verfügen. Grundsätzlich sollten wir uns nicht freiwillig Bedingungen und Faktoren aussetzen, die wir nicht mehr kontrollieren können und der Phantasie eines potentiellen Anspruchstellers ist sowenig Raum wie möglich zu lassen.

\section{Kommunikation}

Um einen Misserfolg der Untersuchung, d.h. spätere Streitigkeiten jedweder Art wirksam zu verhindern, ist eine gute Kommunikation mit dem Auftraggeber zu jeder Phase der Untersuchung am besten wirksam. Durch offene, verständliche und eingehende Information und Beratung kann das Vertravensverhältnis aufgebaut und erhalten werden, das einen später vor Forderungen schützt. Der Klient muss im Vorfeld der Untersuchung durch erklärende Informationen schon vorbereitet werden, er sollte bei der Untersuchung möglichst anwesend und über jeden Untersuchungsgang aufgeklärt sein. Er muss davon überzeugt sein, dass der von ihm gewählte Tierarzt erfahren, kompetent und fähig ist. Er muss Vertraven haben und wissen, was mit dem Pferd geschieht. Er muss auch wissen, wo die Grenzen einer Kaufuntersuchung liegen, d.h. was sie nicht leisten kann und er sollte sich schriftlich damit einverstanden erklären. Eine vertrauensvolle Kommunikation ist jedoch auch mit dem Verkäufer notwendig. Dieser muss in Betracht ziehen, dass eine gründliche Untersuchung möglicherweise Befunde ergibt, die er nicht kennt, von denen er jedenfalls nicht wissen will und die den Wert seines Pferdes vielleicht einschränken. Tierarzt und Auftraggeber müssen mit diesen Situationen umgehen können. Der Verkäufer kennt in der Regel dieses Risiko und wird auch deshalb eine eher nicht so gründliche Untersuchung seines Pferdes wünschen. Je weiter wir aber diesem Wunsch nachgeben, desto mehr begeben wir uns auf Glatteis und in eine riskante Haftungssituation. Gerade jetzt, unter den Regularien des neven Schuldrechts müssen wir den Verkäufer davon überzeugen, dass eine möglichst gründliche Untersuchung des Pferdes ganz in seinem Sinne ist. Er muss sich darüber hinaus mit allen diagnostischen Eingriffen im Verlauf der Untersuchung einverstanden erklären und Tierarzł und Käufer bereitwillig über Daver des Besitzverhältnisses, vorangegangene Medikationen, Operationen, Unfälle, Verhaltensabweichungen, Untugenden oder andere Besonderheiten informieren und er muss schriftlich bestätigen, dass er hierüber nach bestem Wissen Auskunft gegeben hat.

\section{Die Rahmenbedingungen}

Mantell hat die Rahmenbedingungen der Kaufuntersuchung wie folgt zusammengefasst:

- Angemessene Präsentation des Pferdes

- klare Informationen an, und offene Kommunikation mit Käufer und Verkäufer

- geeignete Einrichtung und Umgebung für alle Untersuchungsgänge

- ausreichende Erfahrung und technische Ausrüstung für eine gründliche Untersuchung

- Erkennung von der Norm abweichender Befunde

- Unterscheidung zwischen krankhaften Befunden und Normvarianten

- Bewertung der Befunde im Zusammenhang mit der geplanten Verwendung des Pferdes

- Einschätzung der Notwendigkeit zusätzlicher Untersuchungen

- Entwicklung eines sicheren Gefühls für "foul play" und routinemäßige Entnahme einer Dopingprobe

- vollständige Untersuchung

- lückenlose Aufzeichnung und verständliche Erläuterung der Befunde

- Abgabe einer klaren zusammenfassenden schriftlichen Bewertung und Beratung, die dem Käufer zu einer sicheren Kaufentscheidung verhilft, sich jedoch prognostischer Beurteilungen enthält.

\section{Erweiterung des Standards}

Die Kaufuntersuchung ist zunächst eine klinische Untersuchung mit bisher standardisiertem Minimalumfang. Inwieweit die Untersuchungsteile 1-4 des Protokolls erweitert werden, soll die Diskussion beim Pferdeheilkunde Forum 2002 Mai ergeben. Zur Disposition stehen in diesem Zusammenhang 10 Punkte, die in der Folge kurz abgehandelt werden sollen.

- Schriftliche Erklärung des Verkäufers zur Vorgeschichte des Pferdes

- Standardisierung der Augenuntersuchung

- Obligatorische Dopingprobe

- Endoskopie der Atemwege

- Rektale Untersuchung

- Untersuchung des Rückens

- Zangenpalpation der Hufe 
- Longieren auf hartem Zirkel

- Belastung unter dem Reiter

- Wiederholung des Vortrabens und der Beugeproben nach der Belastung

\section{Schriftliche Erklärung des Verkäufers}

Schon vor Beginn der Untersuchung soll künftig eine schriftliche Erklärung des Verkäufers über die Vorgeschichte des Pferdes vorliegen, die dieser nach bestem Wissen verfasst. Danach liegen dem Käufer und dem untersuchenden Tierarzł spätestens zum Zeitpunkt der Untersuchung die folgenden Informationen vor: Daver des bisherigen Besitzverhältnisses, Ausbildungs- und Trainingsstand, frühere Unfälle, Erkrankungen, Lahmheiten, Operationen, Medikationenen, wann zuletzł und weshalb, Überempfindlichkeiten, Verhaltenstörungen, Haltung, Fütterung, Einstreu, letzte Impfungen und Entwurmungen sowie letzter Beschlag. An dieser Stelle soll sich der Verkäufer schriftlich auch mit allen diagnostischen Maßnahmen und Eingriffen im Zusammenhang mit der Kaufuntersuchung, insbesondere mit einer möglichen Endoskopie und/oder rektalen Untersuchung, der Entnahme einer Dopingprobe, dem Longieren des Pferdes auf hartem Boden, der Untersuchung unter dem Reiter sowie dem Abnehmen der Eisen im Falle einer Röntgenuntersuchung der Strahlbeine einverstanden erklären. Dieser schrifflichen Erklärung kommt bei der Kaufuntersuchung künftig eine zentrale Bedeutung zu. Mit ihr soll der Risikotransfer vom Verkäufer zum Tierarzt teilweise wieder umgekehrt und nebenbei auch das Negativimage des Pferdehandels verbessert werden. Sie stellt so eine wichtige Maßnahme zur Risikoreduzierung dar.

\section{Augenuntersuchung}

Die Untersuchung der Augen soll künftig bezüglich Untersuchungsgang und der technischen Hilfsmittel standardisiert werden, um ein bestmögliches Ergebnis zu gewährleisten. Dabei ist die Untersuchung in einem abgedunkelten Raum und die Verwendung eines Ophthalmoskops absolute Bedingung. Steht ein solcher Raum nicht zur Verfügung, kann die Untersuchung nicht vorgenommen werden und deshalb sollte das Vorhandensein optimaler Untersuchungsbedingungen immer schon im Vorfeld der Untersuchung abgeklärt werden. In den meisten Fällen dürfte die spontane Stellung der Pupille nicht ausreichen, um das Augeninnere eingehend zu untersuchen. Deshalb ist die Verwendung eines Mydriatikums eigentlich unabdingbar. Der Besitzer muss darauf hingewiesen werden, dass die medikamentöse Weitstellung der Pupille Teil der vollständigen Untersuchung ist. In dieser Hinsicht ist auch der Ablauf der Gesamtuntersuchung zu modifizieren, d.h. die Untersuchung des Auges ist an den Schluss zu stellen. Nach Abschluss der Belastungsprüfung, an der Longe oder unter dem Reiter, nach Beruhigung des Pferdes und nach Absolvierung der zweiten orthopädischen Untersuchung kann das Pferd für eine mögliche Endoskopie leicht sediert und für die Ophthalmoskopie mydiatisiert werden, ohne dass diese Maßnahmen den Untersuchungsablauf stören würden.

\section{Dopingprobe}

Die Entnahme einer Blutprobe zum möglichen Nachweis einer Medikation vor der Untersuchung soll künftig obligatorisch sein und zwar weitgehend unabhängig vom Wert des Pferdes. Man kann wohl davon ausgehen, dass die Verführung, ein Pferd für die Kaufuntersuchung fit zu spritzen, noch größer ist als vor dem Turniereinsatz. Wird jedoch obligatorisch eine Dopingprobe genommen, finden diese sicherlich gängigen betrügerischen Machenschaften ein Ende. Es geht dabei nicht in erster Linie darum, den potentiellen Falschspieler zu überführen, sondern um den erzieherischen Faktor. Der zeitliche und finanzielle Mehraufwand für Entnahme, Verarbeitung und Lagerung ist angesichts der weitreichenden Vorteile zu vernachlässigen. Es erscheint ausreichend, eine Blutprobe zu nehmen und diese für eine noch festzulegende Frist tiefgekühlt aufzubewahren. Die relativ hohen Kosten der chemischen Analyse der Probe fielen nur im Verdachtsfall an und dann werden sie auch gerne bezahlt. Verwendung finden soll ein fälschungssicherer Kit, der in Anwesenheit des Auftraggebers verschlossen und beschriftet wird und, wie dies von den FEl-Dopingproben bekannt ist, nur im Fachlabor geöffnet werden kann.

\section{Endoskopie der Atemwege}

Die klinische Untersuchung der oberen und unteren Atemwege soll künftig häufiger durch die Endoskopie nach der Belastungsuntersuchung ergänzł werden. Die lange Liste obskurer Atemwegserkrankungen, die nur durch eine Routineuntersuchung zu erkennen sind, ist beeindruckend. Zu diskutieren ist hier jedoch der Umfang der Untersuchung, d.h. die Frage ob die Luftsackspiegelung mit einbezogen werden soll oder nicht. Der diagnostische Wert der Endoskopie besonders im Hinblick auf den Kehlkopf und den COB-Status ist unstrittig und auf das Plus, mögliche versteckte Befunde aufzudecken, sollte künftig nicht mehr verzichtet werden. Schon heute endoskopieren viele Kollegen bei Kaufuntersuchungen regelmäßig und einer Einführung in den Routinestandard steht eigentlich nichts im Wege. Ich denke, Tierärzte die Kaufuntersuchungen durchführen, werden auch ein Endoskop besitzen, sodass nicht davon auszugehen ist, dass mit dieser Maßnahme eine außerordentliche finanzielle Belastung verbunden ist. Die Untersuchung kann in leichter Sedation am Ende der Kaufuntersuchung vor oder nach der Augenuntersuchung stattfinden, ohne den Gesamtablauf zu behindern.

\section{Rektaluntersuchung}

Ist es sinnvoll, das Pferd im Zuge der Kaufuntersuchung auch rektal zu untersuchen? Handelt es sich um ein Zuchttier, so wird die Maßnahme sowieso durchgeführt. Aber jeder Tierarzt weiß, dass häufig genug Zufallsbefunde gestellt werden, die ohne rektale Untersuchung nicht hätten entdeckt werden können. Chronische Dickdarm-Obstipationen z.B. sind unter den modernen Haltungs- und Fütterungsbedingungen nicht selten und wenn sie anlässlich der Kaufuntersuchung erkannt werden, kann das Pferd entsprechend umgestellt werden, ohne dass dies die Kaufentscheidung beeinflussen muss. Auch hier gilt, dass wir durch eine gründliche Untersuchung ohne viel Mehraufwand das diagnostische Spektrum erweitern und unser Ansehen als verantwortungsbewusste Tierärzte erhöhen.

\section{Untersuchung von Hals und Rücken}

Die gründliche klinische Untersuchung des Rückens nimmt etwas mehr Zeit in Anspruch und wird schon aus diesem Grunde bisher 
gerne vernachlässigt. Dabei kann sie bei der äußeren Begutachtung von Gebäude und Stellung sowie bei der Beurteilung der Körperoberflächen ohne großen Aufwand mit erledigt werden. Dies wird üblicherweise nach dem ersten Untersuchungsgang noch in der Box oder nach dem Herausführen des Pferdes auf die Vorführstrecke durchgeführt. Bei der visuellen und palpatorischen Begutachtung der Hautoberfläche wird nun ergänzend besonderes Augenmerk auf die Hals- und Rückenpartie gerichtet, diese von allen Seiten besichtigt, die Körperseiten verglichen und die Provokationsproben möglichst nach einem Standardverfahren angeschlossen. Gerade im Zusammenhang mit diesem Untersuchungsteil wird auch die Notwendigkeit einer Begutachtung des Pferdes unter dem Reiter klar. Wie wertvoll ist es, das Pferd beim Aufsatteln und Aufsitzen zu beobachten und wie viel Informationen über Rückenprobleme erhält der Tierarzt durch direkte Beobachtung des gerittenen Pferdes und durch Informationen des Reiters bei einer Belastung des Pferdes in der ihm zugedachten Disziplin. Nur so können Taktunreinheiten oder mangelnde Durchlässigkeit erkannt werden, die durchaus auch medizinische Ursachen im Bereich von Hals und Rücken haben können.

\section{Hufzange}

Viele Kollegen untersuchen bei der Kaufuntersuchung die Hufe auch mit der Hufzange, aber längst nicht alle und auch nicht in jedem Fall. Diese aufschlussreiche diagnostische Maßnahme könnte künftig obligatorisch sein. Natürlich müssen die Ergebnisse vorsichtig bewertet werden, da die individuelle Druckempfindlichkeit der Pferde entsprechend der jeweiligen Hufkonstitution sehr unterschiedlich ist. Trotzdem sind lokale Schmerzbereiche oder eine abnorme Empfindlichkeit in der Strahlbeinregion durch nichts besser zu erkennen als durch eine sorgsame Zangenpalpation der Hufe.

\section{Longieren auf hartem Zirkel}

Die Mehrzahl aller Ansprüche nach Kaufuntersuchungen, ca. 40\%, betrifft Lahmheiten. Das heißt, dass gerade in diesem Bereich ein Mehr an Diagnostik dringend notwendig ist. In diesem Sinne erscheint es angebracht, das Pferd obligatorisch auf hartem Boden zu Longieren. Das Longieren auf hartem Zirkel wird zwar kontrovers diskutiert, jedoch nur wegen angeblicher Verletzungsgefahr, wenn zu eng oder auf rutschigem Boden longiert wird. Unter optimalen Bedingungen ist der diagnostische Wert dieser Maßnahme jedoch unstrittig, denn unter dieser besonderen Belastung treten auch undeutliche Lahmheiten zu Tage. Wird darauf geachtet, dass der Zirkel ausreichend groß und rutschfest ist, dann sollte dagegen nichts einzuwenden sein. In den Fällen, in denen der Besitzer des Pferdes diesen Test nicht genehmigt, sollte dies künftig im Protokoll vermerkt werden.

\section{Untersuchung unter dem Reiter}

Eine ähnlich wertvolle Erweiterung des diagnostischen Spektrums ermöglicht uns die Belastung des Pferdes unter dem Reiter und die damit verbundene Gelegenheit auch auf das Vorhandensein orthopädischer Probleme der Gliedmaßen zu achten. Mögliche Erkrankungen des Rückens und manche Lahmheit sind in der Reitbahn deutlicher zu erkennen. Der rationelle Untersuchungsablauf gestattet es, während dieser 10 bis 15 Minuten mehrere Punkte zu erledigen: Belastungsprüfung, die Herzauskultation nach
Belastung, Beurteilung der Atmung, Einschätzung möglicher Atemgeräusche, Begutachtung von Hals, Rücken und Gängen und Beurteilung möglicher Lahmheiten oder Gangstörungen, die besonders unter dem Reiter offensichtlich werden.

\section{Zweites Vortraben und Wiederholung der Beugeproben}

Mit diesem Untersuchungsteil im Zusammenhang steht das sich nun anschließende wiederholte Vortraben an der Hand auf hartem Boden sowie die Wiederholung aller Beugeproben. Es gibt Lahmheiten, die kurz nach dem Herausführen des Pferdes aus der Box offensichtlich sind. Es gibt aber auch die, welche erst durch Belastung deutlich werden. Statistisch belegen lässt sich das nicht, aber dass dem so ist, weiß jeder Tierarzt. Diese zweite Begutachtung des Pferdes an der Hand bietet im Übrigen auch die Gelegenheit, ein zweites Mal über das ganze Tier zu sehen und vielleicht Befunde zu erkennen, die man bei der ersten Untersuchung übersehen haben mag.

\section{Röntgenuntersuchung}

Diese bisher genannten 10 Punkte betreffen die Erweiterung der klinischen Kaufuntersuchung der bisherigen Abschnitte 1-4 des Protokolls. Es schließt sich nun in vielen Fällen, meist abhängig von Alter und Preis des Pferdes, eine Röntgenuntersuchung an, deren Wert allerdings nicht immer so klar ist, wie es zu wünschen wäre. Röntgenbilder können die Kaufentscheidung nicht nur erleichtern, sie erschweren diese leider auch oft genug. Vielleicht weil ihnen zuviel Bedeutung zugemessen wird? Eine gute Lösung ist es, die Entscheidung für eine ergänzende radiologische Untersuchung nicht nur von Alter und Wert des Pferdes, sondern auch vom Ergebnis der klinischen Untersuchung abhängig zu machen. Ist die Röntgenuntersuchung Teil des Auftrags, was zweifellos immer häufiger der Fall ist, dann muss das Standardprogramm, welches sich ja doch immer auf einen Kompromiss reduziert, ganz ohne Frage vollständig sein. Den Minimalstandard legt in diesem Frühjahr die Röntgenkomission neu fest. Nicht dazu gehören wahrscheinlich die Tangentialaufnahmen der Strahlbeine, die Zehen der Hintergliedmaßen seitlich, die 4 Schrägaufnahmen der vorderen Fesselgelenke bzw. Gleichbeine und die 3. Sprunggelenksebene. Es wäre jedoch wünschenswert, dass diese wichtigen zusätzlichen Aufnahmen mehr und mehr routinemäßig angefertigt werden. Bei unseren Nachbarn in der Schweiz und in den Niederlanden z.B. ist diese Verfahrensweise üblich und es erscheint sinnvoll, sich anzupassen und gleiche Methoden einzuführen. Aufnahmen der Sattellage, Kniegelenksaufnahmen oder andere Bilder werden nach Absprache mit dem Auftraggeber oder bei Verdacht ergänzend angefertigt und bei außerordentlich teveren Pferden oder in Verdachtsfällen können auch besondere Untersuchungen wie die Szintigraphie, CT oder Sonographie angezeigt sein. Besonders bei der Befundung der Röntgenbilder, die vorzugsweise unter optimalen Lichtbedingungen und im "stillen Kämmerlein" durchgeführt werden soll, ist die eingehende und verständliche Beratung des Auftraggebers sehr wichtig und prognostische Bewertungen sind gerade hier tunlichst zu unterlassen.

\section{Haftung}

Wie bereits vorher schon erwähnt, kommt der guten Kommunikation zwischen Tierarzt und Auftraggeber bei der Kaufuntersu- 
chung zentrale Bedeutung zu. Dies gilt auch für die allgemeinen Informationen bezüglich der Haftung. Der Auftraggeber muss über die Haftungssituation vollständig aufgeklärt sein. Dies betrifft ausdrücklich nicht die rechtlichen Bedingungen des Pferdekaufs. Hier halten wir uns gänzlich zurück und überlassen die nicht einfache Aufgabe den Juristen. Der Auftraggeber der Kaufuntersuchung muss aber wissen, weshalb z.B. die Untersuchung so eingehend und umfangreich protokolliert wird, warum z.B. der Tierarzt auf einer Haftungsfrist von 6 Monaten besteht oder weshalb er keine Gewährleistung für äußere Fehler des Pferdes, Verhaltensbesonderheiten, insbesondere Koppen oder für Allergien übernehmen kann. Wird ein Pony im Winter untersucht, dann können wir keine Aussagen über ein mögliches Sommerekzem machen und kommt ein Pferd von der Weide zur Kaufuntersuchung, dann kann eine COB-Empfänglichkeit bei Aufstallung nicht ausgeschlossen werden.

Die für den Pferdehandel unter manchen Aspekten eher rückschrittliche Novellierung bzw. Europäisierung des Schuldrechts hat bei den Tierärzten Unsicherheit und Verwirrung gestiftet. Bisher war die Sache für den Tierarzt erfreulich klar. Er haftete für seine gutachterliche Tätigkeit ab dem Zeitpunkt der Untersuchung 6 Monate lang und damit hat es sich gehabt. Leider ist damit jetzt Schluss. Die Haftungsfrist beginnt jetzt erst zum Ende des Jahres, in dem der Mangel erkannt wurde, ist also doch erheblich länger. Doch ist die Verschlechterung der rechtlichen Situation des Tierarztes vielleicht doch nicht ganz so erheblich. Es steht die Tatsache dagegen, dass sich der Käufer eines Pferdes im Falle eines Mangels unter den neven gesetzlichen Regelungen zunächst am Verkäufer schadlos halten wird. Wir stehen hier nicht mehr wie bisher in der direkten Schusslinie. Über die Allgemeinen Vertragsbedingungen können wir heute die Haftungsfrist jedoch nur noch auf 12 Monate beschränken. Da uns dies nach wie vor zu lang erscheint, versuchen wir seit Anfang dieses Jahres, die 6Monatsfrist durch Nebenabrede in Form einer individualvertraglichen Vereinbarung zwischen Tierarzt und Auftraggeber zu regeln. Diese ist ausdrücklich nicht Teil des Untersuchungsvertrages. Es kann nur ein Versuch sein, die Sache in unserem Sinne zu regeln. Wie die Gerichte damit umgehen, bleibt abzuwarten.

Niemand kann derzeit beurteilen, wie die Rechtsprechung mit den für den Pferdehandel unsinnig und unpraktikabel erscheinenden gesetzlichen Bedingungen umgehen wird. Wir müssen jedoch der Entwicklung voraus sein, was den Untersuchungsumfang angeht, den Maßstab festlegen und sowohl für die Klientel als auch für die Gerichte die Rahmenbedingungen vorgeben. Betrachten wir den heutigen, seit 15 Jahren praktizierten Standardumfang der Kaufuntersuchung, dann müssen wir erkennen, dass er angesichts der auf uns zukommenden Erwartungen der Auftraggeber und auch angesichts eines möglichen vermehrten Risikos nicht mehr ausreicht, um uns optimal abzusichern. Die Kaiserliche Verordnung schützte in der Vergangenheit nicht nur den Käufer, sondern sie gab auch dem Tierarzt klare Entscheidungskriterien an die Hand, jedenfalls in Bezug auf chronische Augen-, Kehlkopf- und Lungenerkrankungen. Jetzt ist der Verkäufer in der unangenehmen Situation, 1 bzw. 2 Jahre lang für alle möglichen Mängel des Pferdes zu haften und es ist nur zu natürlich, dass er dieses Risiko durch die tierärztliche Kaufuntersuchung teilen oder am besten ganz an den Tierarzt abgeben will. Um uns vor diesem Missbrauch zu schützen, müssen wir einerseits den Auftraggeber durch schriftliche Erklärung in die Pflicht nehmen und andererseits eingehender untersuchen. Der Minimalstandard ist in Rich- tung Optimalstandard zu verändern. Es ist nicht nur sinnvoll und für alle Beteiligten zumutbar, sondern geradezu unumgänglich, künftig den Standardumfang der Kaufuntersuchung dort zu erweitern, wo es der Sache und zur Vorbeugung rechtlich gefährlicher Situationen des Tierarztes dienlich ist. Die zusätzliche Durchführung der hier vorgeschlagenen ergänzenden klinischen Untersuchungen würde einen zeitlichen Mehraufwand von ca. 30 Minuten bedeuten und es ist selbstverständlich, dass sich dies auch in der Liquidation niederschlagen muss. Der Mehraufwand ist sorgsam gegen den Informationsgewinn abzuwiegen. Die Realisierung jedes einzelnen der vorgeschlagenen 10 Punkte sichert jedoch unsere Position und verbessert unsere Reputation.

Am grundsätzlichen Gebrauch eines Untersuchungsprotokolls mit Vertragscharakter kann es keinen Zweifel mehr geben. Es beinhaltet die grundsätzliche Aufklärung des Auftraggebers über Inhalt, Ablauf und Rahmenbedingungen der Kaufuntersuchung, regelt die Verkürzung der Haftungsfrist, dient der Aufzeichnung der vorgeschichtlichen Informationen und der Identifizierung des Pferdes, erfasst die Ergebnisse aller Untersuchungen, stellt sicher, dass keine Untersuchungsschritte vergessen wurden, enthält die schriftliche Erfassung aller besonderen Befunde, deren Bewertung sowie das Gesamturteil und die Beratung des Auftraggebers und hat als Ganzes Vertragscharakter. Insgesamt soll es gewährleisten, dass der Auftraggeber bestmöglich beraten wird und verhindern, dass der Tierarzt, wie David. W. Jagger es ausdrückt, unvorbereitet in ein juristisches Minenfeld läuft.

Die Neuauflage des Vertrags wird die Erweiterung der Standarduntersuchung berücksichtigen und es wird ein Formblatt für die schriftliche Erklärung des Verkäufers zum Pferd beigelegt, der damit dem Tierarzł gegenüber in die rechtliche Pflicht genommen wird. Ein gesonderter Befundbogen dient der Protokollierung der Atemwegsendoskopie, gleich ob sie zur Standarduntersuchung erklärt oder nur empfohlen wird. Der Vertrag wird nach wie vor einzeln nummeriert und die Nummerierung soll identisch mit der der Dopingprobe sein. Er liegt wieder in anspruchsvoller äußerer Form vor, die dem Besonderen von Zweck und Inhalt gerecht wird. Es ist mehr als ein TÜVBericht, eine Bezeichnung die dem hohen Anspruch und $\mathrm{Ni}$ veau tierärztlicher Diagnostik nicht gerecht wird und dem Auftraggeber auch noch suggeriert, es handele sich um das, was es eben gerade nicht ist, nämlich den Zustandsbericht eines gebrauchten technischen Sportgeräts. Gerade jetzt, wo eine unsensible und in mancher Hinsicht ignorante Gesetzgebung das Tier wieder versachlicht, muss diese Bezeichnung getilgt werden. Hat der Gesetzgeber schon versäumt, die Vorgaben aus Brüssel im Rahmen seines nationalen gesetzgeberischen Spielraums durch Sonderregelungen den besonderen ethischen und praktischen Bedingungen des Tierhandels im Allgemeinen und des Pferdehandels im Besonderen anzupassen, was ja durchaus möglich gewesen wäre, so sollten wir die nun bestehenden Gesetze im Rahmen unserer Möglichkeiten und in unserem Sinne ausfüllen. Ein erster Schritt in diese Richtung ist die Optimierung des Vertrags über die Untersuchung eines Pferdes.

Dr. Hans D. Lauk

Albrechtstraße 11

10117 Berlin

hdlauk@pferdeheilkunde.de 